\title{
Pulmonary Function in Obese individuals
}

\author{
Saumy Johnson*, Luin Jose and Carol Mathews \\ Department of Respiratory Therapy Sciences, Inaya Medical College, Saudi Arabia
}

Submission: June 18, 2019; Published: June 26, 2019

*Corresponding author: Saumy Johnson, Assistant professor and Vice Chair, Department of Respiratory Therapy Sciences, Inaya Medical College, Riyadh, Kingdom of Saudi Arabia

Abstract

This review tries to discuss the researches that are related to pulmonary function in obesity, its mechanisms and consequences. Abdominal adiposity could be considered as a better predictor of pulmonary function than weight or BMI. Obesity has important effects on respiratory function, which add considerably to the load of respiratory disease. Weigh loss is found to improve lung function in patients with respiratory disorders especially with asthma.

Keywords: Obesity; Pulmonary function; Abdominal adiposity; Weight; BMI; Weight loss; Asthma; Respiratory disorders; Fat accumulation; Noncommunicable diseases; Endometrial; Breast; Ovarian; Prostate; Liver; Gallbladder; Kidney; Colon

Abbreviations: WHO: World Health Organization; DM: Diabetes Mellitus; ILs: Interleukins; WC: Waist Circumference; FVC: Forced Vital Capacity; AOR: Adjusted Odds Ratio; DH: Dynamic Hyperinflation; COPD: Chronic Obstructive Pulmonary Disease

\section{Introduction}

World Health Organization (WHO) defines overweight and obesity as abnormal or excessive fat accumulation that presents a risk to health. Overweight is a BMI greater than or equal to 25 ; and obesity is a BMI greater than or equal to 30 . The worldwide prevalence of obesity almost tripled between 1975 and 2016. Higher BMI is a major risk factor for noncommunicable diseases such as: cardiovascular diseases (mainly heart disease and stroke), which were the leading cause of death in 2012, diabetes; musculoskeletal disorders (especially osteoarthritis - a highly disabling degenerative disease of the joints), Malignancies (including endometrial, breast, ovarian, prostate, liver, gallbladder, kidney, and colon) [1].

Pulmonary dysfunction has been shown in type 2 diabetes mellitus (DM)[1]. Yeh HC et al. reported decreased lung function is independently associated with Metabolic syndrome and with Diabetes Mellitus, and impaired lung function presents before the development of MS or DM; these associations may result from the effects of obesity and inflammation [2,3]. The pulmonary dysfunction in DM may not be of much clinical interest because of the effect size, Yet, for the reason that of the relationship with other conditions such as obesity, smoking and heart failure, and the resulting high morbidity and mortality due to reduced lung function, as well as in the context of developing new inhaled insulin products, it is serious that there be a enhanced understanding of the relation between lung function and diabetes and the relationship of this connection $[3,4]$.
Leone $\mathrm{N}$ et al. [5] reported that abdominal obesity and poor lung function are associated with a low-grade inflammatory state, which might contribute to metabolic disease and ill-health. High levels of interleukins (ILs) 6 and 8, tumor necrosis factor $\alpha$ (TNF- $\alpha$ ), CRP, leptin, and lower levels of adiponectin, which helps in regulating insulin sensitivity, are all reported. In respiratory disorders like OSA, OHS, or COPD with release of adipocytokines affecting systemic inflammation perhaps triggered by hypoxemia induced by obesity and adipose tissue is now regarded as an endocrine organ [6].

Abnormality in the values of FEV1, FVC and FEV1/FVC was linked with the components of the metabolic syndrome, most importantly with abdominal obesity and with elevated low-density lipoproteins, hypertension, and insulin resistance. They were independent of age, sex, BMI, history of cardiovascular diseases, smoking, or alcohol use. In this research the PFT measurements were all restrictive lung pattern, which is usually seen in obesity-related lung changes [5].

Ochs-Balcom HM et al. [7] reported that in women, abdominal height and waist circumference were negatively associated with FEV (1) percent predicted, while adiposity markers were negatively associated with FVC percent predicted. In men, all overall and abdominal adiposity markers were inversely associated with FEV (1) percent predicted and FVC percent predicted. Abdominal adiposity could be considered as a better predictor of pulmonary 


\section{Current Research in Diabetes \& Obesity Journal}

function than weight or BMI, and researchers should consider it when investigating the determinants of pulmonary function.

Saxena Y et al. [8] in their study on abdominal obesity and pulmonary functions in young Indian adults of random sample of volunteers $(n=80)$ from general population in and around Dehradun, India of age group 20-40 years found out that in women the waist circumference(WC) shows a higher negative association to respiratory parameters FVC and FEV1 $\{\mathrm{B}$ (P value), R2 as $-0.381(0.017), 0.122$ and $-0.373(0.019), 0.139\}$ while all the adiposity markers showed a negative significant association. In men WC showed highly significant negative association with FVC and FEV1 \{Beta (P value), R2 as - 0.502(0.001), 0.232 and $0.428(0.006), 0.184\}$ with higher R2 values as compared to other adiposity parameters. The result suggested that the abdominal obesity marker is an important and better predictor of pulmonary function than BMI and the investigators suggest the inclusion of it as a potential confounding factor when investigating the determinants of pulmonary function.

A study by Pan J et al. [9] assessed adiposity indices including BMI, waist circumference (WC), waist hip ratio, waist height ratio and body fat percentage. Lung function was assessed by spirometry using a turbine flow meter. They analyzed percent predicted for forced expiratory volume in $1 \mathrm{~s}$ (FEV1\%), forced vital capacity (FVC \%) and restrictive respiratory defect (FEV1/FVC ratio > low limits of normal and FVC \% <0.80). There were 16186 participants ( mean age $61.4 \pm 7.2$ years; $74.0 \%$ women), Comparing to those with neither central nor general obesity, those with only central adiposity and with both central and general obesity had lower pulmonary function (adjusted $\beta$ range from -2.85 to -6.02 for FEV1\% and FVC\%, adjusted OR range from 1.14 to 1.70 , all $\mathrm{P}$ $<0.05$ ). Those with only general obesity had better but non-significant pulmonary function. (Crude $\beta$ range from 1.46 to 2.92 for FEV1\% and FVC\%, crude OR range from 0.68 to 0.93 , all P > 0.05). A positive association of central or general obesity with restrictive respiratory defect was observed (adjusted odds ratio (AOR) from 1.50 to 2.04, all $\mathrm{P}<0.002$ ).These findings suggest that (central adiposity WC: $\geq 90 \mathrm{~cm}$ in men, $\geq 80 \mathrm{~cm}$ in women) and its indices, but not general adiposity and BMI, were independently associated with lower pulmonary function and higher risk of restrictive respiratory defect in older Chinese individuals.

\section{Lung Function After Bariatric Surgery and Weight Loss}

Wei YF studied Pulmonary function and anthropometric factors were studied in 94 obese patients aged 18-65 years with a body mass index $>32 \mathrm{~kg} / \mathrm{m}$ (10). Pulmonary function tests were performed preoperatively and 3 months after bariatric surgery. The changes in anthropometric parameters were analyzed in relation to pulmonary function test results. Multiple linear regression models were applied to identify the factors that influenced pulmonary function after bariatric surgery. When measured 3 months after surgery, all anthropometric parameters for the 94 patients studied had significantly decreased, and the pulmonary function test parameters had significantly improved. Of the anthropometric parameters, the reduction in body weight, WC, and waist/height ratio correlated significantly with increases in the FEV (1) and FVC. In the multiple linear regression analysis, only the reduction in WC correlated significantly with the reductions in the FEV (1) and FVC. All the anthropometric parameters of obesity decreased significantly and the pulmonary function improved after the surgery. This improvement correlated best with the reduction in the WC and perhaps a decreased intra-abdominal pressure [10].

Twenty-four women with morbid obesity were evaluated by Campos EC et al. [11] with regard to lung function, respiratory muscle strength, functional capacity, body composition, anthropometrics and the level of habitual physical activity two weeks before and six months after bariatric surgery. It was found that body composition and anthropometric variables exerted a direct influence on functional capacity and lung function in the women analyzed but exerted no influence on sedentary life style, even after accentuated weight loss following bariatric surgery.

Moderate weight loss can improve dynamic hyperinflation (DH) in obese patients with asthma that is associated with a decrease in abdominal fat. Furthermore, a minimum of $5 \%$ in weight loss delays the onset of DH and expiratory flow limitation besides inducing a clinical improvement in asthma quality of life and clinical control [12]. Weight loss may be beneficial for improving asthma control in overweight and obese patients [13].

\section{Mechanism Behind the Lung Dysfunction}

Impact of obesity on respiratory system may give added problems, changing the balance of pressures between the inward recoil of the lung and the outward pressure of the chest. This has major effects on lung volumes, airway mechanics, airway closure, expiratory flow limitation, ventilation distribution, airway hyper responsiveness, and exhaled nitric oxide levels. Many of the changes in lung function due to obesity are likely to contribute to respiratory symptoms. Yet there are significant differences in the effect of obesity on lung function between children, adolescents, and adults. Hence understanding the effects of obesity on lung function throughout the lifespan is essential to understanding present and future effects of obesity on respiratory health [14].

Obesity has important effects on respiratory function, which add considerably to the load of respiratory disease. These mechanical effects are not readily quantified with usual pulmonary function testing and measurement of body mass index. Changes in mediators formed by adipose tissue probably also add to altered lung function, although so far this is inadequately understood [15].

Obese individuals have impaired respiratory function relative to their normal-weight counterparts. Despite these negative effects, obesity is paradoxically associated with better survival in individuals with chronic obstructive pulmonary disease (COPD). Obese COPD patients have reduced operating lung volumes and higher inspiratory capacity to total lung capacity ratios than their 
lean COPD counterparts. Obese patients with COPD do not appear to be at a disadvantage during exercise relative to lean COPD patients. Obesity may be associated with improved survival in COPD but specific mechanisms for this paradox remain to be elucidated [16].

\section{References}

1. https://www.who.int/en/news-room/fact-sheets/detail/obesity-and-overweight

2. Yeh HC, Punjabi NM, Wang NY, Pankow JS, Duncan BB, et al. (2008) Cross-sectional and prospective study of lung function in adults with type 2 diabetes: The Atherosclerosis Risk in Communities (ARIC) study. Diabetes Care 31(4): 741-746.

3. Klein OL, Krishnan JA, Glick S, Smith LJ (2010) Systematic review of the association between lung function and type 2 diabetes mellitus. Diabet Med 27(9): 977-987.

4. Fawn Yeh, Anne E Dixon, Susan Marion, Carl Schaefer, Ying Zhang, et al. (2011) Obesity in Adults Is Associated with Reduced Lung Function in Metabolic Syndrome and Diabetes: The Strong Heart Study. Diabetes Care 34(10): 2306-2313.

5. Leone N, Courbon D, Thomas F, Bean K, Jégo B, et al. (2009) Lung function impairment and metabolic syndrome. The critical role of abdominal obesity. Am J Respir Crit Care Med 179(6): 509-516.

6. Franssen FME, O’Donnell DE, Blaak EE, Schols AMWJ (2008) Obesity and the lung: 5. Obesity and COPD. Thorax 63(12): 1110-1117.

7. Ochs-Balcom HM, Grant BJ, Muti P, Sempos CT, Freudenheim JL, et al. (2006) Pulmonary function and abdominal adiposity in the general population. Chest 129(4): 853-862.

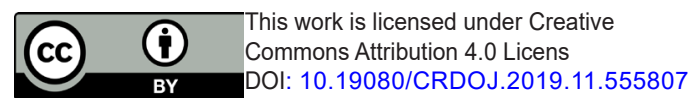

8. Saxena Y, Sidhwani G, Upmanyu R (2009) Abdominal obesity and pulmonary functions in young Indian adults: a prospective study. Indian J Physiol Pharmacol 53(4): 318-326.

9. Pan J, Xu L, Lam TH, Jiang CQ Zhang WS, et al. (2017) Association of adiposity with pulmonary function in older Chinese: Guangzhou Biobank Cohort Study. Respir Med 132: 102-108.

10. Wei YF, Tseng WK, Huang CK, Tai CM, Hsuan CF, et al. (2011) Surgically induced weight loss, including reduction in waist circumference, is associated with improved pulmonary function in obese patients. Surg Obes Relat Dis 7(5): 599-604.

11. Campos EC, Peixoto-Souza FS, Alves VC, Basso-Vanelli R, Barbalho-Moulim M, et al. (2018) Improvement in lung function and functional capacity in morbidly obese women subjected to bariatric surgery. Clinics (Sao Paulo) 73: e20.

12. Aline Grandi Silva, Patricia Duarte Freitas, Palmira Gabriele Ferreira, Rafael Stelmach, Regina Maria Carvalho-Pinto (2019) Effects of weight loss on dynamic hyperinflation in obese women asthmatics. J Appl Physiol 126(2): 413-421.

13. Adeniyi FB, Young T (2012) Weight loss interventions for chronic asthma. Cochrane Database Syst Rev (7): CD009339.

14. David Chapman, Gregory King, Erick Forno (2019) Obesity and lung function: From childhood to adulthood Mechanisms and Manifestations of Obesity in Lung Disease. 978-0-12-813553-2, Academic Press, Elsevier Inc, USA.

15. Anne E Dixon, Ubong Peters (2018) The effect of obesity on lung function. Expert Rev Respir Med 12(9): 755-767.

16. Jordan Guenette, Dennis Jensen, Denis O’Donnell (2010) Respiratory function and the obesity paradox. Curr Opin Clin Nutr Metab Care 13(6): 618-624.

\begin{tabular}{l} 
Your next submission with Juniper Publishers \\
will reach you the below assets \\
- Quality Editorial service \\
- Swift Peer Review \\
- Reprints availability \\
- E-prints Service \\
- Manuscript Podcast for convenient understanding \\
- Global attainment for your research \\
- Manuscript accessibility in different formats \\
( Pdf, E-pub, Full Text, Audio) \\
- Unceasing customer service \\
Track the below URL for one-step submission \\
https://juniperpublishers.com/online-submission.php \\
\hline
\end{tabular}

